\title{
Phase-contrast tomography of neuronal tissues: from laboratory- to high resolution synchrotron CT
}

\author{
Mareike Töpperwien, Martin Krenkel, Kristin Müller, and Tim Salditt \\ Institute for X-Ray Physics, Friedrich-Hund-Platz 1, Göttingen, Germany
}

\begin{abstract}
Assessing the three-dimensional architecture of neuronal tissues with sub-cellular resolution presents a significant analytical challenge. Overcoming the limitations associated with serial slicing, phase-contrast x-ray tomography has the potential to contribute to this goal. Even compact laboratory CT at an optimized liquid-metal jet microfocus source combined with suitable phase-retrieval algorithms and preparation protocols can yield renderings with single cell sensitivity in millimeter sized brain areas of mouse. Here, we show the capabilities of the setup by imaging a Golgi-Cox impregnated mouse brain. Towards higher resolution we extend these studies at our recently upgraded waveguide-based cone-beam holo-tomography instrument GINIX at DESY. This setup allows high resolution recordings with adjustable field of view and resolution, down to the voxel sizes in the range of a few ten nanometers. The recent results make us confident that important issues of neuronal connectivity can be addressed by these methods, and that 3D (virtual) histology with nanoscale resolution will become an attractive modality for neuroscience research.
\end{abstract}

Keywords: x-ray phase-contrast tomography, 3D virtual histology, in-line holography, waveguide-based imaging, laboratory-based imaging

\section{INTRODUCTION}

Understanding the three-dimensional cytoarchitecture and connectivity of neuronal tissue represents a major challenge in neuroscience research as it plays an essential role in the task of fully understanding the brain. Methods like histological sectioning, ${ }^{1}$ light sheet fluorescence microscopy ${ }^{2}$ magnetic resonance imaging ${ }^{3}$ or serial block-face electron microscopy ${ }^{4}$ are the scientific standard but each method faces its own challenges like invasive sample preparation or lack of contrast or resolution. x-ray computed tomography is a promising approach for deciphering the cytoarchitecture and connectivity of the brain in a non-invasive manner as the small wavelength and high penetration depth enable a high three-dimensional resolution in relatively large specimens. Classical computed tomography relies on the absorption of the x-rays while passing through the sample. This leads to sufficient contrast for highly absorbing structures like the bones but only yields a low contrast for weak absorbing soft tissue like the brain. The real part of the x-ray index of refraction $n(\mathbf{r})=1-\delta(\mathbf{r})+i \beta(\mathbf{r})$, the decrement $\delta$, gives rise to a a sample-induced phase shift and is up to 1000 times larger for hard x-rays and soft tissue than the extinction coefficient $\beta$ leading to the absorption of the beam. Therefore the phase shift instead of the absorption should be exploited for contrast formation. As it is not possible to measure the phase of the x-ray beam directly, this phase shift has to become visible. This can ,e.g., be achieved via Zernike-based phase contrast, ${ }^{5}$ Talbot interferometry, ${ }^{6,7}$ edge-illumination ${ }^{8,9}$ or speckle-based phase contrast. ${ }^{10}$ For high resolution reconstructions contrast formation by free space propagation behind the object ${ }^{11-13}$ is a well suited method enabling a resolution in the range of a few ten nanometers in two dimensions. ${ }^{14}$ Interference of the disturbed wavefronts leads to characteristic intensity modulations in the detector plane which are then used to reconstruct the phase information of the object with suitable phase-retrieval algorithms. ${ }^{15}$

Due to the high degree of coherence needed for the interference of the disturbed wavefronts, propagation-based phase-contrast imaging was long believed to be a sole synchrotron-based technique. However, with the invention of new x-ray microfocus sources, delivering a relatively high flux while simultaneously providing a sufficiently

Further author information: (Send correspondence to M.T. or T.S.)

M.T.: mtoeppe@gwdg.de

T.S.: tsaldit@gwdg.de

Developments in X-Ray Tomography X, edited by Stuart R. Stock, Bert Müller, Ge Wang, Proc. of SPIE Vol. 9967, 99670T · (c) 2016 SPIE · CCC code: 0277-786X/16/\$18 · doi: 10.1117/12.2238496 
high degree of partial coherence, this imaging technique can also be employed at laboratory setups.

Here we present propagation-based phase-contrast tomography of mouse neuronal tissue stained with the GolgiCox impregnation technique to get high contrast within the tissue in order to resolve the structure of single neurons as well as the connectivity between selected cells in 3D. We investigate the sample at our laboratory setup consisting of a liquid-metal jet microfocus source and compare it to measurements taken at the P10 beamline at DESY (Hamburg), showing that sub-cellular resolution in relatively large brain samples is possible at both setups and that even issues of connectivity might be answered by combining the synchrotron setup with suitable sample preparation and phase-retrieval algorithms.

Phase-retrieval algorithms In order to get quantitative reconstructions of the three-dimensional density distribution in the sample, the integrated phase information of the object in one projection image has to be reconstructed from the measured intensity images. To this end, two phase-retrieval algorithms are applied in the following, both imposing different requirements on the sample.

For weak objects and small propagation distances $z$ between the object and the detector, the transport of intensity equation (TIE) can be linearized, leading to an intensity distribution $I_{z, \theta}\left(\mathbf{r}_{\perp}\right)=I_{0, \theta}\left(\mathbf{r}_{\perp}\right)\left[1-\frac{z}{k} \nabla_{\perp}^{2} \phi_{\theta}\left(\mathbf{r}_{\perp}\right)\right]$ in the detector plane ${ }^{15}$ (with $I_{0, \theta}\left(\mathbf{r}_{\perp}\right)$ and $\phi_{\theta}\left(\mathbf{r}_{\perp}\right)$ being the intensity and phase distribution directly behind the object). Due to the Laplacian relationship between the phase information of the object and the measured intensity image, phase contrast typically appears in this so-called direct contrast regime as edge-enhancement. In Fourier space it can be inverted via a filter of the form $-\frac{1}{\left|\mathbf{k}_{\perp}\right|^{2}}$ with $\mathbf{k}_{\perp}=\left(k_{x}, k_{y}\right)$ being the lateral twodimensional coordinate vector in reciprocal space. To account for the singularity at $\left|k_{\perp}\right|=0$ and weak residual absorption, a regularization parameter $\alpha$ can be added to the denominator of the filter, leading to the so-called Modified Bronnikov Algorithm (MBA) for phase retrieval. ${ }^{16}$ For weak absorbing objects this algorithm provides sharp quantitative reconstructions whereas for objects with non-negligible absorption the reconstructions get blurred. In this case the approximated phase $\phi_{\theta}^{\dagger}\left(\mathbf{r}_{\perp}\right)$, obtained using the MBA scheme, can be used to get a sharp reconstruction of the intensity distribution directly behind the object via

$$
I_{0, \theta}\left(\mathbf{r}_{\perp}\right)=\frac{I_{z, \theta}\left(\mathbf{r}_{\perp}\right)}{1-\gamma \nabla_{\perp}^{2} \phi_{\theta}^{\dagger}\left(\mathbf{r}_{\perp}\right)}
$$

with the $\alpha$-dependent regularization parameter $\gamma$. This reconstruction scheme is known as the Bronnikov Aided Correction $(B A C) .{ }^{17}$

For larger propagation distances the imaging regime shifts to the holographic region where phase contrast is visible in multiple fringes appearing around the object. In this case an algorithm based on the contrast transfer function $(C T F)$ can be used. The CTF is valid for objects showing a weak absorption and a slowly varying phase, ${ }^{18}$ assumptions which are often fulfilled by biological specimens. According to the CTF the intensity distribution in reciprocal space is given by $\tilde{I}\left(\mathbf{k}_{\perp}\right)=2 \pi \delta\left(\mathbf{k}_{\perp}\right)+2 \sin \chi \cdot \tilde{\phi}\left(\mathbf{k}_{\perp}\right)-2 \cos \chi \cdot \tilde{B}\left(\mathbf{k}_{\perp}\right)$, with $\chi=\frac{\lambda z\left|\mathbf{k}_{\perp}\right|^{2}}{4 \pi}$ and $\phi\left(\mathbf{r}_{\perp}\right)=-k \int \delta(\mathbf{r}) \mathrm{d} z$ and $B\left(\mathbf{r}_{\perp}\right)=k \int \beta(\mathbf{r}) \mathrm{d} z$ being the changes in phase and amplitude due to the decrement and extinction coefficient of the refractive index. For objects with a negligible absorption $(B \ll \phi)$ this equation can further be simplified by ignoring the last term. According to the CTF the $\sin \chi$-function acts as a filter for the phase in reciprocal phase, leading to a loss of information for specific spatial frequencies due to the zero crossings of the sine function. In order to be able to reconstruct the phase without artifacts, intensity images at different propagation distances can be recorded to account for these frequencies. ${ }^{11,19}$ Via a least square minimization a reconstruction formula for the phase information of an object with negligible absorption can be obtained: ${ }^{11,19}$

$$
\tilde{\phi}_{\theta}\left(\mathbf{k}_{\perp}\right)=\frac{\sum_{m} \sin \chi_{m} \tilde{I}_{z_{m}, \theta}\left(\mathbf{k}_{\perp}\right)}{\sum_{m} 2 \sin ^{2} \chi_{m}+\alpha} .
$$

The additional frequency-dependent regularization parameter $\alpha\left(\mathbf{k}_{\perp}\right)$ is added to account for remaining zeros of the denominator. Due to the different nature of the zero crossings at $\left|\mathbf{k}_{\perp}\right|=0$ and $\left|\mathbf{k}_{\perp}\right|>0$ the regularization parameter is defined differently for high and low spatial frequencies.

Even for objects with non-negligible weak absorption a reconstruction formula can be found. Under the assumption of a homogeneous object with $\phi\left(\mathbf{r}_{\perp}\right)=-\frac{\delta}{\beta} B\left(\mathbf{r}_{\perp}\right)$ the phase distribution of the object in reciprocal space 


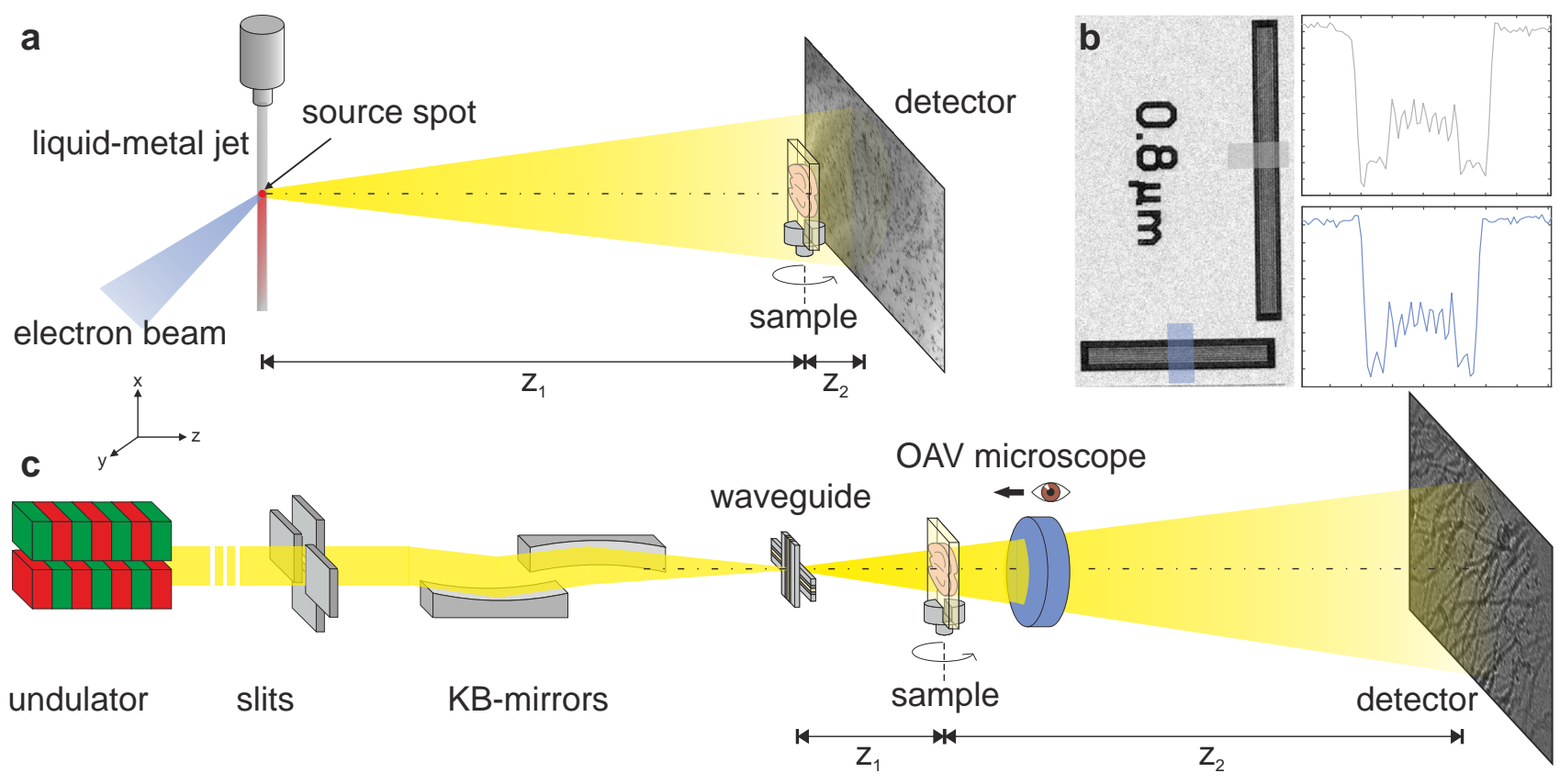

Figure 1. Experimental setups. a Schematics of the laboratory setup. The x-rays are generated by a liquid-metal jet source, enabling a small source spot in combination with a relatively high flux, and directly pass the sample without any further optical elements. Some distance behind the object the detector is recording the modulated intensity pattern. $\mathbf{b}$ Image of an absorbing test pattern showing a resolution of $800 \mathrm{~nm}$ in $2 \mathrm{D}$, both in horizontal and vertical direction. c Schematics of the GINIX setup at the P10 beamline at DESY (Hamburg). The x-rays are generated by an undulator and, after several beam shaping elements as well as a $\mathrm{Si}(111)$ channel-cut monochromator, focussed by a Kirkpatrick-Baez (KB) mirror system. A waveguide is placed into the focus of the KB mirrors to further minimize the effective source size, to generate a smooth illumination and to increase the coherence of the beam. Behind the waveguide the sample is placed, followed by a detector recording the intensity image approximately $5 \mathrm{~m}$ behind the sample.

can be retrieved via ${ }^{18}$

$$
\tilde{\phi}_{\theta}\left(\mathbf{k}_{\perp}\right)=\frac{\sum_{m} C_{m} \mathcal{F}_{\perp}\left[I_{z_{m}, \theta}\left(\mathbf{r}_{\perp}\right)-1\right]}{\sum_{m} 2 \cdot C_{m}^{2}+\alpha}
$$

with $C_{m}=\sin \chi_{m}+\frac{\beta}{\delta} \cos \chi_{m}$ and $\mathcal{F}_{\perp}$ being the two-dimensional lateral Fourier transform previously denoted as .

\section{EXPERIMENTAL SETUP}

\subsection{Laboratory Setup}

In the laboratory setup (see Fig. 1a), the x-rays are generated by a liquid-metal jet source (Excillum, Stockholm, Sweden), providing the necessary partial coherence of the beam by allowing for small spot sizes while at the same time delivering a higher flux compared to conventional x-ray sources. The anode material is galinstan, an alloy consisting of gallium, indium and tin, which is liquid at room temperature and has its peak energy at $9.25 \mathrm{keV}$. Without any further beam-shaping optics the sample is positioned directly behind the exit window of the source at a variable distance $z_{1}$ to the source spot. It is mounted on a sample tower with three translational and one rotational axes which allow for tomographic measurements of the desired part of the sample. Additionally, two further translational axes parallel and perpendicular to the beam are mounted underneath the sample tower to vary the distance of the sample to the source and to align the rotation axis with respect to the optical axis. At an adjustable distance $z_{2}$ behind the object, the detector is placed on a motorized detector stage with two translations perpendicular to the optical axis which allow for the alignment of the pitch angle and the zero position of the rotation. 
Due to the cone-beam geometry of the setup, a high resolution of the imaging system can either be achieved by choosing a high magnification $M\left(z_{1} \ll z_{2}\right)$ leading to a small effective pixel size $d x_{\text {eff }}=\frac{d x}{M}$ of the detector or by choosing a low magnification $\left(z_{1} \gg z_{2}\right)$ and implementing a high resolution detector instead. ${ }^{20}$ In the first case the resolution is limited by the source spot dimensions whereas in the last case the detector PSF is the limiting factor. As the minimal spot size of the source lies in the range of $4 \mu \mathrm{m}$ we chose the second approach in order to obtain the highest possible resolution. For this purpose, the lens-coupled single-crystal scintillator-based CCD camera XSight (Rigaku, Prague, Czech Republic) with a pixel size of $540 \mathrm{~nm}$ has been installed in the setup. This leads to an actual resolution of $800 \mathrm{~nm}$ in 2D, as qualitatively determined by a test pattern (Fig. $1 b)$.

\subsection{GINIX setup at the P10 beamline at DESY}

To achieve higher resolution, additional measurements have also been performed with synchrotron radiation. To this end, the GINIX endstation ${ }^{21-23}$ installed at the P10 beamline at DESY in Hamburg, was used (Fig. 1c). The x-rays are generated by an undulator and after several beam defining components including a $\operatorname{Si}(111)$ channel-cut monochromator focussed by a Kirkpatrick-Baez (KB) mirror system to a size of about $300 \times 300 \mathrm{~nm}^{2}$. To further optimize the illumination with respect to shape, coherence and focal spot, a waveguide is placed into the KB focus. ${ }^{24,25}$ The effect of the waveguide on the illumination is illustrated in Fig. 2. The 2D waveguide system is realized by two crossed planar multilayer waveguides with lengths $l_{1}$ and $l_{2}$, respectively, each with a guiding layer of carbon with width $d=81 \mathrm{~nm}$ (see Tab. 1). Using the waveguide as source for the holographic cone-beam imaging, higher resolution can be achieved than with just the KB beam, at the cost of smaller flux. However, progress in waveguide fabrication ${ }^{25}$ along with a choice of larger guiding layer width $d$ compared to ${ }^{24,25}$ has resulted in a fairly high exit flux (see Tab. 1), enabling short acquisition times. Moreover, as can be seen in the farfield images with (Fig. 2c) and without (Fig. 2b) the waveguide, the illumination is significantly smoothed and does not show the wavefront distortions (stripe pattern) typical for KB farfields. This facilitates the empty-beam correction. ${ }^{26}$

The sample is positioned on a motorized tower at a distance $z_{1}$ behind the waveguide. The sample tower has two translational and one rotational axes for tomographic measurements, as well as two translational and one rotational axes to align the rotation axis with respect to the optical axis, to change the waveguide-sample distance $z_{1}$ and to align the pitch angle of the rotation axis. The position of the sample with respect to the beam can be aligned with an on-axis-view (OAV) microscope. The detector is placed at a distance $z_{2}$ of approximately
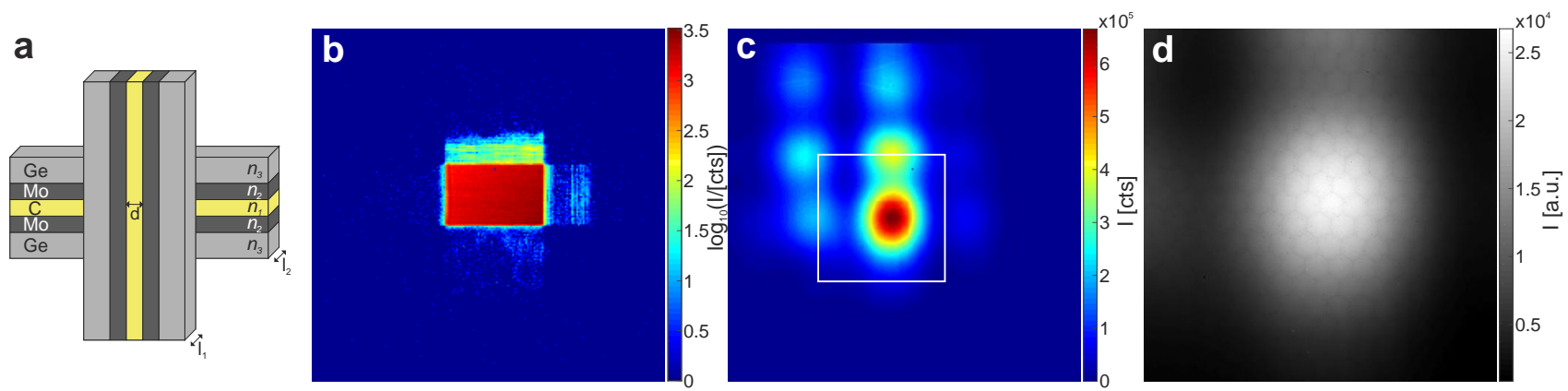

Figure 2. Influence of the waveguide on the shape of the illuminating wavefront. a Schematic of the crossed $2 \mathrm{D}$ waveguide system. It consists of two planar multilayer waveguides of length $l_{1}$ and $l_{2}$ with a carbon guiding layer of thickness $d$. They are combined in a crossed manner to form a $2 \mathrm{D}$ waveguide device. $\mathbf{b}$ Farfield pattern without the waveguide imaged with a Pilatus $300 \mathrm{k}$ with only the central $200 \times 200$ pixels displayed. The rectangular shape reflects the geometric acceptance of the KB mirrors. The shape is determined by geometrical optics. The exposure time was $0.1 \mathrm{~s}$ and a 300 $\mu \mathrm{m}$ thick Mo foil was added for attenutation of the beam. c Farfield pattern with the waveguide installed, imaged in the same experimental configuration, recorded with an exposure time of $1 \mathrm{~s}$ and no additional attenuation. The maximum is diffraction broadened and appears more Gaussian shaped. The typical aberrations produced by the KB mirror system (stripe artifacts) are not observed. The white rectangle marks the approximate field of view of the high resolution sCMOS camera (Hamamatsu) used for imaging. d Image of the illumination obtained with the high resolution sCMOS camera. 
Table 1. Parameters of the used waveguides.

\begin{tabular}{lccccc}
\hline & energy & length $l_{1}$ & length $l_{2}$ & width $d$ & flux \\
\hline Fig. 2,3,5 & $13.8 \mathrm{keV}$ & $368 \mu \mathrm{m}$ & $320 \mu \mathrm{m}$ & $81 \mathrm{~nm}$ & $3 \cdot 10^{9} \mathrm{ph} / \mathrm{s}$ \\
Fig. 8\&9 & $13.8 \mathrm{keV}$ & $480 \mu \mathrm{m}$ & $357 \mu \mathrm{m}$ & $81 \mathrm{~nm}$ & $9 \cdot 10^{8} \mathrm{ph} / \mathrm{s}$ \\
\hline
\end{tabular}

$5 \mathrm{~m}$ behind the focal plane. In between, a flight tube prevents absorption of the x-rays in air.

Images are recorded by a sCMOS camera with a fibre-coupled scintillator, converting the x-rays to visible light. In the case of the Epon embedded sample, the detector was equipped with a $20 \mu \mathrm{m}$ single crystal LuAG:Ce scintillator (Hamamatsu, Tokyo, Japan). In the case of the hydrated samples (stained and unstained), a camera with a $15 \mu \mathrm{m}$ Gadox scintillator was used (Photonic Science, Sussex, UK). The pixel size of both detectors is $6.5 \mu \mathrm{m}$. Based on the cone-beam geometry, the pixel size transforms via the geometrical magnification $M=\frac{z_{1}}{z_{1}+z_{2}}$ to a demagnified effective pixel size $d x_{\text {eff }}=\frac{d x}{M}$.

\subsection{Sample preparation}

\subsubsection{Golgi-Cox stained brain slices}

A wild type mouse was anesthetized with $\mathrm{CO}_{2}$ and perfused with $4 \%$ para-formaldehyde (PFA) to ensure tissue preservation. Afterwards the brain was dissected and directly prepared for tissue staining following the FD Rapid GolgiStain ${ }^{\mathrm{TM}}$ Kit (FD NeuroTechnologies, Inc., Columbia, USA). Prior to the last step in the staining procedure, the brain was cut into $1 \mathrm{~mm}$ thick slices and afterwards either embedded in Epon (Serva, Heidelberg, Germany) or stored in PBS until the measurements. For the Epon embedding, the samples were first washed with PBS and $\mathrm{H}_{2} \mathrm{O}$ and subsequently dehydrated in an ascending acetone series (50\% acetone: 5 min, $70 \%$ acetone: 5 min, 90\% acetone: $5 \mathrm{~min}, 100 \%$ acetone: $3 \times 5 \mathrm{~min})$. Afterwards the sample was transferred into Epon (50\% Epon in acetone for $30 \mathrm{~min}, 70 \%$ Epon in acetone for $30 \mathrm{~min}, 90 \%$ Epon in acetone for $30 \mathrm{~min}, 100 \%$ Epon for $30 \mathrm{~min}$ and over night in an embedding mould). As a final step, the Epon was polymerized at $60{ }^{\circ} \mathrm{C}$ for $24 \mathrm{~h}$. For the measurement, the Epon block was cut around the edges of the sample to minimize absorption of the x-rays.

For the hydrated samples, a liquid chamber was built based on two aluminum rings with a diameter of $5 \mathrm{~cm}$ and a width of $500 \mu \mathrm{m}$ which were covered with polypropylene foil on one side. Two of those rings were then glued together to build a chamber, after putting the brain slice as well as a small amount of PBS on one of the foils (see Fig. 8a and b). The orientation of the aluminum rings was chosen in such a way that the resulting chamber width equals the thickness of the brain slice. In this way, the sample was held in place by the pressure of the two polypropylene foils.

\subsubsection{Unstained brain slice}

A wild type mouse was anesthetized with $\mathrm{CO}_{2}$ and perfused with $10 \%$ sucrose solution for 10 minutes. Afterwards the brain was dissected and fixed over night with $1 \%$ para-formaldehyde and $1 \%$ glutaraldehyde. The fully fixed brain was cut into slices of $500 \mu \mathrm{m}$ and $1 \mathrm{~mm}$ thickness and stored in PBS until the measurement. The sample mounting at the beamline was performed as in the case of the hydrated Golgi-Cox stained brain slices.

\section{RESULTS}

\subsection{Epon embedded Golgi-Cox stained brain slice}

\subsubsection{Laboratory setup}

Figure 3a shows an overview of the sample, recorded at the laboratory setup. The source-sample-distance $z_{1}$ is $158.75 \mathrm{~mm}$ and the sample-detector-distance $22.5 \mathrm{~mm}$, resulting in an effective pixel size of $0.47 \mu \mathrm{m}$. In order to image the whole sample, 15 neighboring projections have been recorded, each with an exposure time of $50 \mathrm{~s}$, and virtually stitched together. For a better signal-to-noise ratio, the projections were resampled by a factor of 2. The neurons are already very well discernible in the the $2 \mathrm{D}$ projections, due to their sparse staining. Their morphology reveals that the brainslice is taken from the region of the hippocampus as this structure is clearly distinguishable. The large red rectangle marks the region of the recorded tomogram at the laboratory setup with a field of view of $1.18 \times 1.57 \mathrm{~mm}^{2}$, whereas the smaller rectangles mark the regions of the measurements 

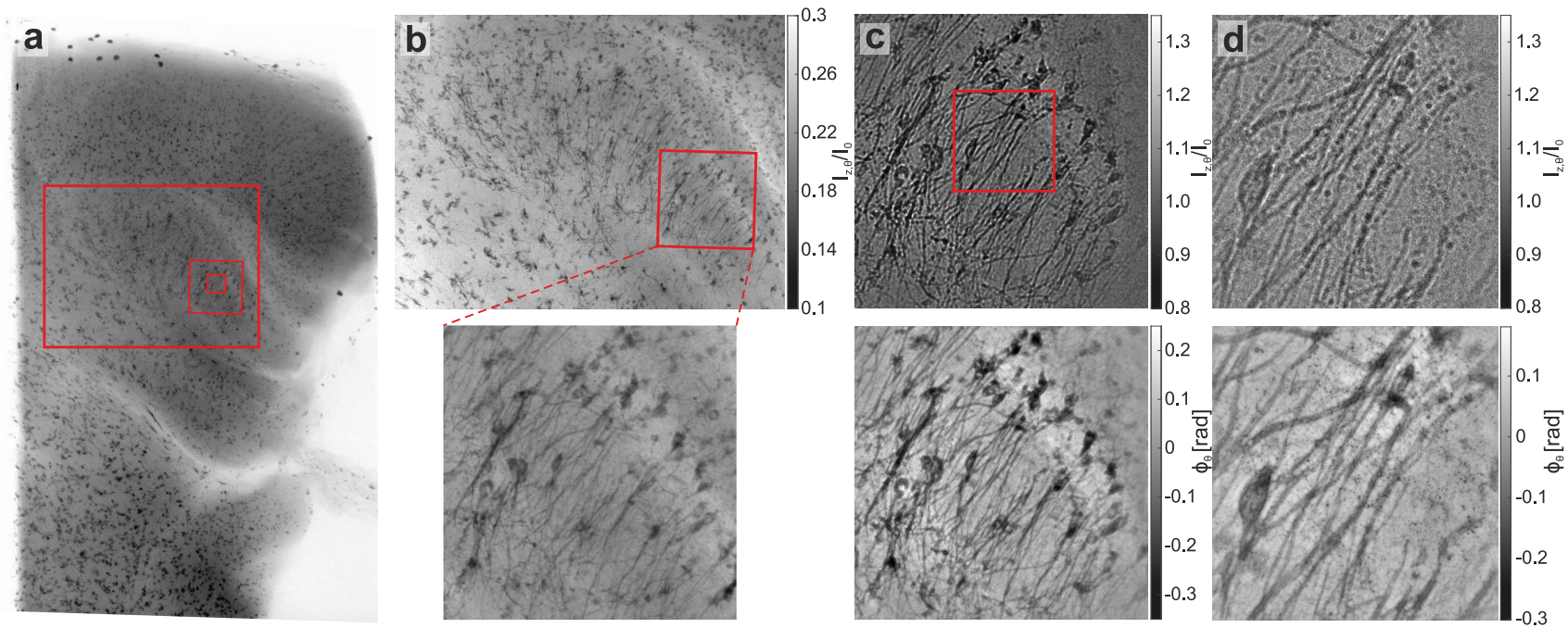

Figure 3. Projection of the Epon embedded Golgi-Cox stained sample obtained with the different experimental configurations. a Overview scan of the sample at the laboratory setup. As the sample is too large for the field of view of the camera 15 projections at different positions of the sample were recorded and later stitched together. The large rectangle marks the position of the tomogram measured at the laboratory setup wheres the two smaller ones mark the positions of the two measurements at the synchrotron. b Flatfield-corrected projection of the tomogram obtained at the laboratory setup. To achieve a better signal-to-noise ratio the projection is resampled by a factor of 2 . As the object is due to the silver mainly an absorbing structure the effect of the phase-retrieval is negligible. The rectangle marks the position of the synchrotron measurement with lower resolution c Flatfield-corrected projection recorded at the synchrotron at a pixel size of $182 \mathrm{~nm}$ (top) as well as the phase obtained by the CTF approach for weakly absorbing objects using four propagation distances. The rectangle marks the position of the zoom measurement. In contrast to the laboratory measurement, phase-contrast effects are clearly visible due to the higher degree of coherence and longer propagation distance. d Projection of the zoom measurement obtained at the synchrotron with a pixel size of $63 \mathrm{~nm}$ (top) and the corresponding phase again reconstructed with the $\mathrm{CTF}$ algorithm from four propagation distances.

at the P10 beamline. One exemplary projection of the laboratory tomogram is depicted in Fig. 3b with an inset showing part of the projection at higher magnification. This part corresponds to the position of the lower resolution tomogram recorded at the P10 beamline, presented in section 3.1.2.

For the tomogram, 2000 projections were recorded over $180^{\circ}$ with an exposure time of $100 \mathrm{~s}$ (see Tab. 2 for a summary of the experimental parameters). Due to the stable sample preparation, these long exposure times should not lead to alterations of the sample during the measurement. The BAC phase retrieval is performed on each projection image with the parameters $\alpha=0.03$ and $\beta=0.15$. The effect of the phase retrieval is however rather small, as the contrast of the object is dominated by absorption due to the large $Z$-value of silver. Tomographic reconstruction is performed with the ASTRA toolbox. ${ }^{27,28}$ One transversal slice through

Table 2. Experimental parameters for the measurements of the Golgi-Cox stained samples measured at the laboratory and the synchrotron.

\begin{tabular}{lcccccc}
\hline & $z_{1}[\mathrm{~mm}]$ & $z_{2}[\mathrm{~mm}]$ & eff. pixel size $[\mu \mathrm{m}]$ & \# distances & \# projections & exp. time $[\mathrm{s}]$ \\
\hline Epon embedded & & & & & & \\
\hline laboratory & 158.75 & 22.5 & 0.47 & 1 & 2000 & 100 \\
P10 overview & 145 & 5040 & 0.182 & 4 & 1000 & 0.4 \\
P10 zoom & 50 & 5135 & 0.063 & 4 & 1000 & 0.2 \\
\hline Hydrated & & & & & & \\
\hline P10 overview & 145 & 5040 & 0.182 & 1 & 1500 & 1 \\
P10 zoom & 65 & 5120 & 0.081 & 1 & 1500 & 1 \\
\hline
\end{tabular}


the reconstructed volume is shown in Fig. 4a. It becomes evident that motion artifacts have occurred which lead to disturbed reconstructions. In view of the stable sample preparation, it is highly likely that these motion artifacts arise from temperature drifts and associated thermal expansion of the specimen as well as its holders made mainly from aluminum. Under the assumption of merely translational movements, however, these artifacts can be corrected for.

Translational motion correction For the vertical motion, the linogram of the tomographic scan can be used for correction. To this end, all projections are horizontally integrated and the resulting profile is plotted against the rotation angle (Fig. 4b). In the absence of vertical movements, this should result in straight horizontal lines. Therefore by aligning the projections to each other, an undistorted linogram is reconstructed and the vertical motion can be determined. The shift for each projection with respect to the first one is shown in Fig. 4f (top) and the reconstructed slice from the vertically corrected projections in Fig. 4c. This first correction already improves the data quality noticeably. To further improve the reconstruction, also the horizontal movement should be corrected for. For this purpose the sinogram is taken into account. As the opening angle of the setup is approximately $0.25^{\circ}$, the influence of the cone-beam geometry on the shape of the sinogram can be neglected.
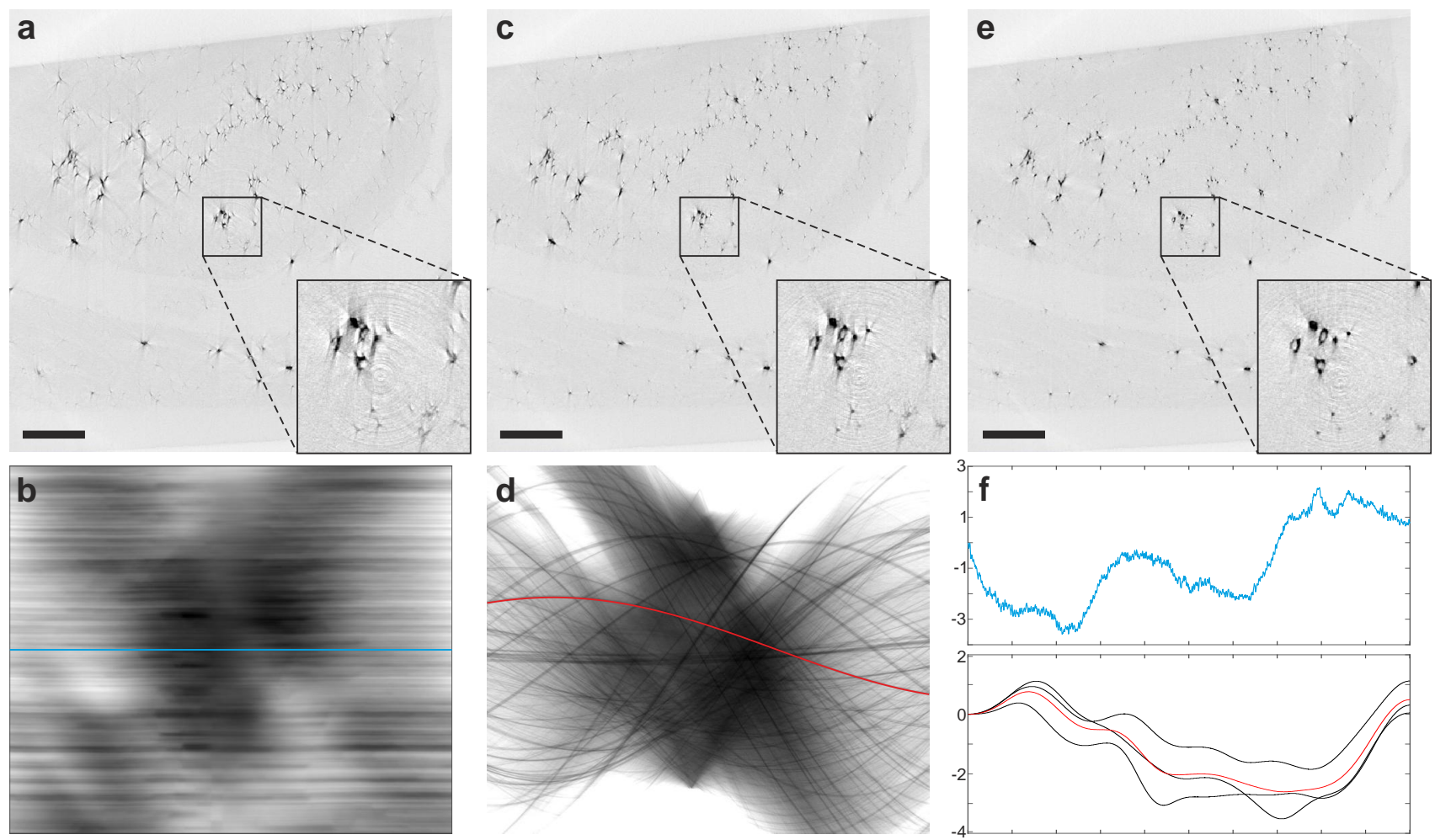

Figure 4. Motion correction in horizontal and vertical direction for the laboratory data. a Reconstructed slice without any motion correction applied. The features within the slice show typical motion artifacts as e.g. features do not appear closed or round objects show streak like artifacts. b Linogram of the tomography scan. To obtain the linogram, all projections are integrated along the $x$-direction and the corresponding profiles are plotted against the rotation angle. Without any vertical movement this should result in straight horizontal lines (as indicated by the blue line). The deviations from this line are shown in $\mathrm{f}$. c Slice reconstructed from the projections which have been corrected for vertical movement. The motion artifacts are clearly reduced. d Exemplary sinogram of the scan. Without any horizontal movement of the sample, the shown curves should follow a perfect sine function. To correct for the motion, one well recognizable curve is selected and a sine is fitted to it. The deviations from the sine function indicate the horizontal movement. For three exemplary curves, the results are shown in f. e Slice obtained after correction with the linogram and the sinogram. The motion artifacts are clearly further reduced. f (Top) Deviations of the linogram from horizontal lines. (Bottom) Deviations of three exemplary curves in the sinogram from the perfect sine function as well as the mean of those (red). Scalebars: 200 $\mu \mathrm{m}$ 
Therefore the single curves of the sinogram should follow a sine function, whereas deviations to the sine function are caused by horizontal movement of the sample. To account for this movement, well recognizable curves in the sinogram are selected based on visual inspection and fitted to a sine (Fig. 4d). The deviations from the sine function are then determined for three different curves (Fig. 4f (bottom, black lines)). The red curve denotes the mean of the three selected curves and the shifts are applied to the projections to account for the horizontal movement. One reconstructed slice from the projections aligned according to both the linogram and the sinogram is shown in Fig. 4e. Motion artifacts are significantly decreased and the objects appear as closed features. The remaining halo around the objects is caused by beam hardening due to the polychromatic illumination and also missing wedge due to the high absorption of the sample in one direction (see the sketch of the sample in Fig. 1).

3D visualization of the laboratory data In Figure 5a a longitudinal slice through the reconstructed volume is shown in which the red rectangles indicate the positions of the two synchrotron measurements at the GINIX setup. Close-ups of these regions can be seen in Fig. 5b and c. The large structures in the slice can be assigned to the cell bodies of the neuronal cells whereas the smaller structures belong to axons or unbound silver molecules.
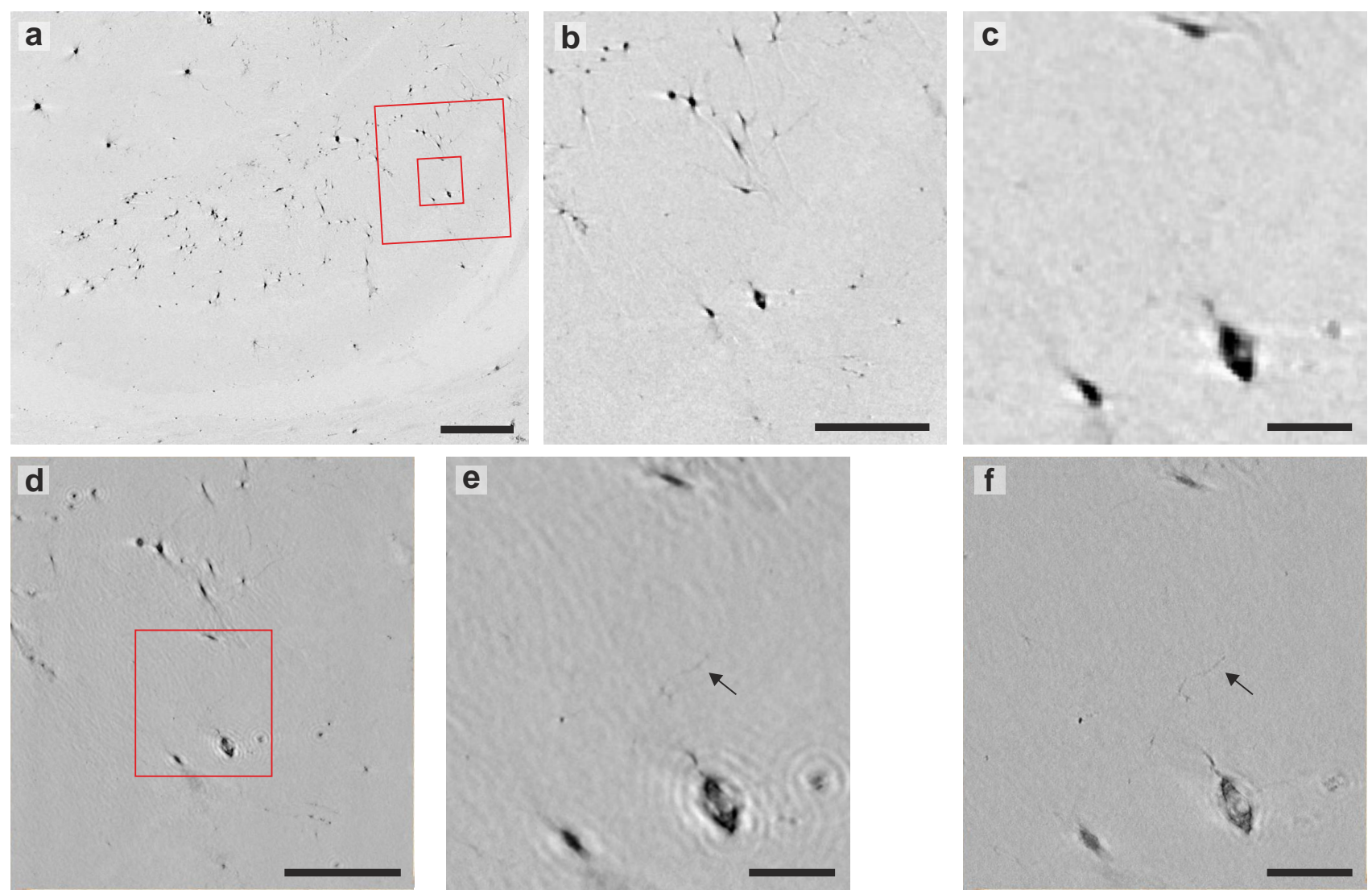

Figure 5. Comparison of reconstructed slices through the Epon embedded sample, obtained at the laboratory setup and at the P10 beamline. a Slice through the reconstructed volume from the laboratory measurement. The rectangles indicate the positions and fields of view of the synchrotron measurements. b Part of the slice from the laboratory showing only the field of view from the lower resolution synchrotron measurement. c Part of the laboratory slice showing the field of view of the high resolution measurement. d Slice through the reconstructed volume of the lower resolution synchrotron measurement. The artifacts which are especially apparent close to the high density features are probably caused by an imperfect phase retrieval and missing wedge. The rectangle marks the field of view of the zoom measurement. e Part of the slice showing the field of view of the zoom measurement. The arrow marks a part of the stained cell which is not resolved in the laboratory measurement. f Slice through the reconstructed volume of the high resolution synchrotron dataset. Compared to the other datasets in $\mathbf{c}$ and $\mathbf{e}$, the structure of the internal silver distribution is resolved in higher detail whereas the coarse shape of the impregnated cell is the same. Scalebars: a $200 \mu \mathrm{m}, \mathbf{b}, \mathbf{d} 100 \mu \mathrm{m}$ and $\mathbf{c}, \mathbf{e}, \mathbf{f} 25 \mu \mathrm{m}$ 
However, due to the small thickness of $0.95 \mu \mathrm{m}$ of the virtual slice the shape of the individual cells cannot be reconstructed directly as the cell emerges over several adjacent slices. To get a better impression of the 3D structure of the cells an automatic gray value based segmentation of the pyramidal cell layer of the hippocampus is shown in Fig. 6a. The segmentation and visualization is performed with the software Avizo (FEI Visualization Sciences Group, Burlington, USA). The rectangle marks a single cell which was also recorded in the two synchrotron measurements. For comparison also a segmentation of this single cell is performed and can be seen in Fig. 6b. The cell body and the axon are clearly resolved. However, in the upper part of the cell it cannot unequivocally be determined whether the observed junction is formed by the cell itself or by a connecting cell which was not stained entirely. For a better visualization of the reconstructed volume see also Video 1.

\subsubsection{GINIX setup}

In order to achieve a higher resolution, corresponding measurements are carried out at the GINIX setup at the P10 endstation at DESY. Due to the cone-beam geometry of the setup, overview scans with a relatively large field of view of $373 \times 373 \mu \mathrm{m}^{2}$ can be combined with zoom measurements of selected regions of interest recorded with a smaller effective pixel size and therefore higher resolution ${ }^{29}$ (see Tab. 2). In Fig. 3c and $d$ exemplary projections of an overview and a zoom scan are shown with a rectangle marking the position of the high resolution measurement with respect to the overview scan. At the top, the raw empty-beam corrected projections can be seen whereas the bottom images show the reconstructed phase using the CTF approach for weakly absorbing objects with four propagation distances taken into account. Each of the 1000 projections per distance taken over $180^{\circ}$ was recorded with an exposure time of $0.4 \mathrm{~s}$ (overview scan) or $0.2 \mathrm{~s}$ (zoom scan), leading to a total measurement time which is at least a factor of 125 (overview) or 250 faster than in the laboratory, not taking the readout time of the cameras into account. However, it should be noted that due to the non-restricted experimental time in the laboratory compared to the synchrotron, the laboratory scan was not optimized for speed but signal-to-noise ratio instead. The reconstruction of the $3 \mathrm{D}$ volume is carried out with the matlab implemented iradon function using the standard Ram-Lak filter. Slices through the volume at
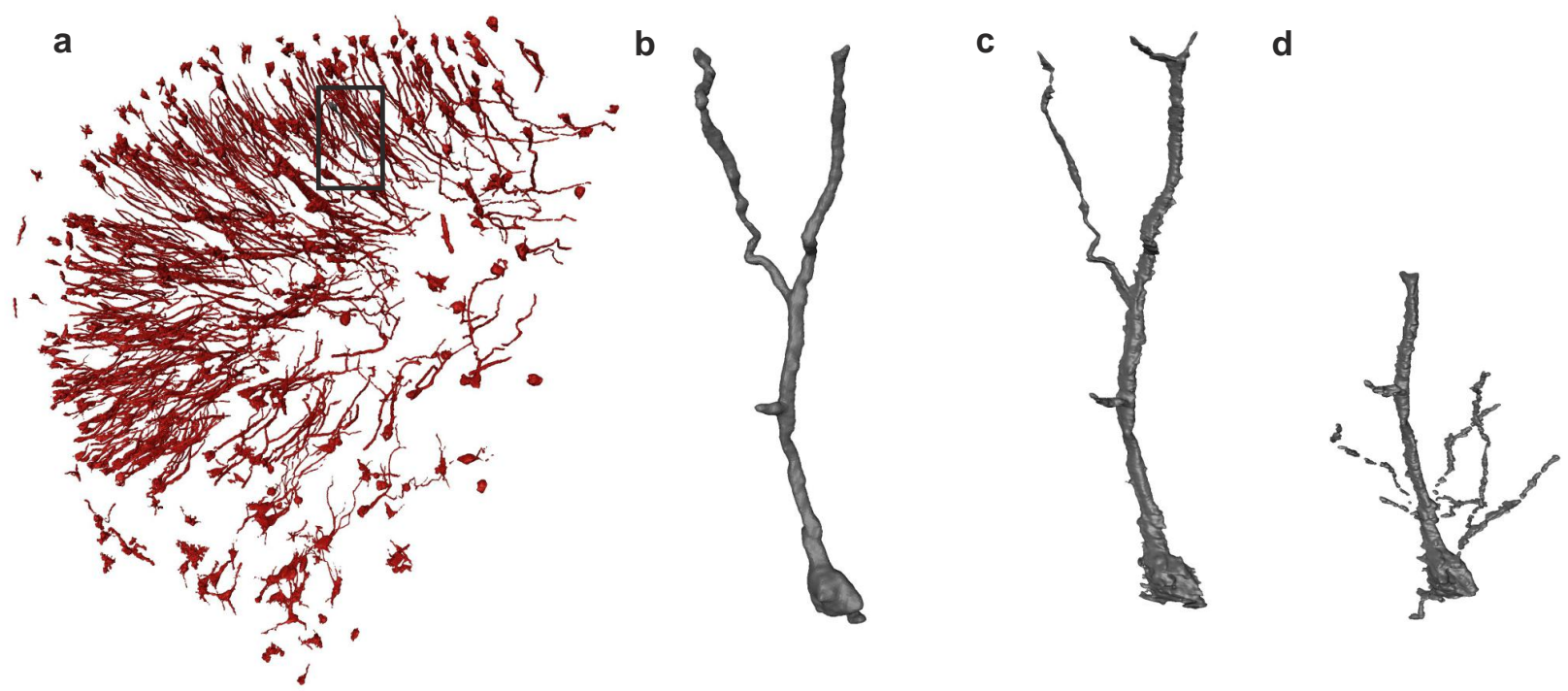

Figure 6. 3D visualization of the Epon embedded sample. a Segmentation of the pyramidal cell layer of the hippocampus in the laboratory data, performed automatically with a gray-value threshold. b Automatic gray value based segmentation of one particular cell (marked with a rectangle in (a)), measured with the laboratory setup. c Automatic segmentation of the same cell in the lower resolution synchrotron scan. The higher resolution compared to the laboratory does not provide much more details concerning the outer structure of the cell. d Semi-automatic segmentation of part of the same cell in the high resolution synchrotron scan. The automatic segmentation also shows the cell in approximately the same detail as the previous two measurements. Only after manual segmentation of structures which do not have a connection but seem to belong to the cells, an advantage of the higher resolution becomes apparent on the level of the 3D shape reconstrution. Note that the missing connection might be caused by incomplete staining. 

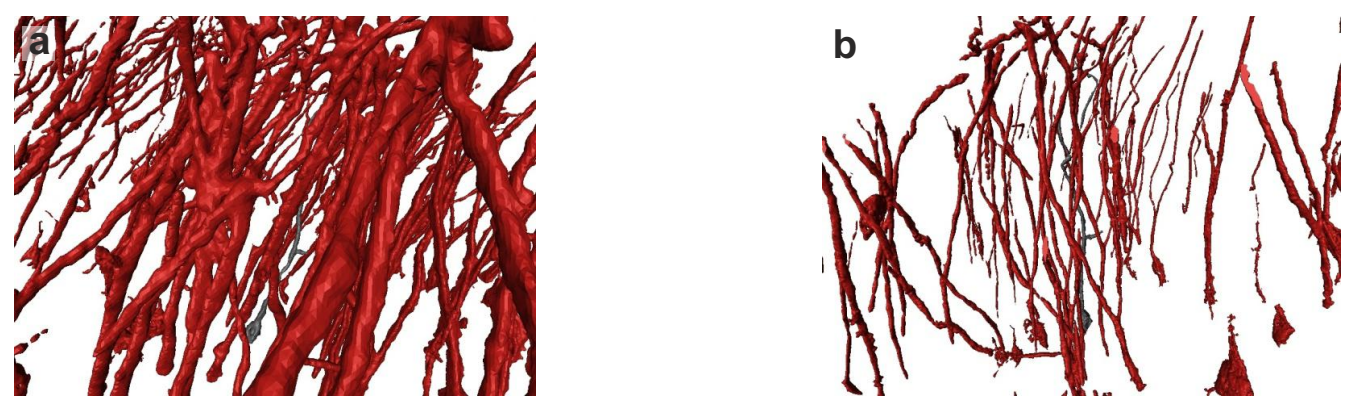

Figure 7. a Video 1: 3D visualization of the Epon embedded sample measured at the laboratory setup. In the beginning, the transverse slices through the volume are shown, followed by an automatic volume rendering of all stained structures and a segmentation of the pyramidal layer of the hippocampus. In the end, the single cell, also measured at the synchrotron, is shown. http://dx.doi.org/10.1117/12.2238496.1 b Video 2: 3D visualization of the Epon embedded sample measured at the synchrotron. For both datasets, the overview scan and the zoom measurement, the transverse slices through the volume are shown as well as a segmentation of the stained cells within this volume. http://dx.doi.org/10.1117/12.2238496.2

equivalent positions are shown in Fig. 5d-f, where e shows the part of the overview scan which corresponds to the field of view of the zoom dataset. Residual fringes are visible in the overview scan and also slightly in the zoom dataset, indicative for imperfect CTF phase retrieval. This can be attributed to the fact that the assumption of a weak object are not completely fulfilled. Further, a white halo around the dense objects (silver) is observed, which is a missing wedge artifact caused by the high absorption of the Epon block in one direction. For a better visualization of the three-dimensional shape of the cells, a (semi-)automatic gray value based segmentation is performed (Fig. 6c and d and Video 2) for the same cell as for the laboratory measurements. In this procedure, stained objects which seem to belong to the cell but have no distinct connection enabling the fully automatic rendering, have been additionally segmented based on visual inspection.

Comparison with the laboratory results Considering the slices through the object shown in Fig. 5, the benefit of the higher resolution measurements at the synchrotron are obvious. Inner cell structures are reconstructed with higher detail and parts of the cell, which are not resolved in the laboratory measurement can now be discerned (see arrows in Fig. 5e and f). However, when considering the segmentation of the single cell, the advantages of the synchrotron measurements become less obvious, as the outer structure of the cell is very similar in Fig. 6b and c. Only by manually adding structures attributed to the cell or adjacent interacting cells, the benefit of the higher resolution becomes evident on the level of the three-dimensional shape reconstruction.

\subsection{Hydrated Golgi-Cox stained brain slice}

As Epon embedding represents a considerable alteration of the sample environment compared to the hydrated state of the brain slice, we have next scanned hydrated Golgi-Cox stained tissues. This preparation state is only suitable for synchrotron measurements, as it is less stable and hence requires significantly shorter scan times. One exemplary projection of the overview scan, again with a field of view of $373 \times 373 \mu \mathrm{m}^{2}$, is shown in Fig. 8c. Phase retrieval has been performed based on single distance CTF, assuming a weakly absorbing object. The position of the overview scan with respect to the whole slice can be seen Video 3. The rectangle in c shows the position of the zoom dataset in d recorded with an effective pixel size of $81 \mathrm{~nm}$. Exemplary empty-beam corrected projections are shown in the top row (unreconstructed images), and the corresponding single distance $\mathrm{CTF}$ reconstructions in the bottom row. Although four propagation distances were measured in the case of the zoom dataset, only the first distance was used for phase retrieval, as a comparison between projections of different distances indicated that the silver had slowly dissipated from the cells. This is probably due to radiation damage. By only using the first propagation distance, this effect could be minimized. Both measurements were carried out with an exposure time of $1 \mathrm{~s}$ per projection and 1500 projections over $180^{\circ}$ (see Tab. 2 for a summary of the experimental parameters).

Longitudinal slices through the reconstructed volume using the matlab implemented function iradon with a standard Ram-Lak filter are shown in Fig. 9a and b. For comparison both slices are generated at approximately 

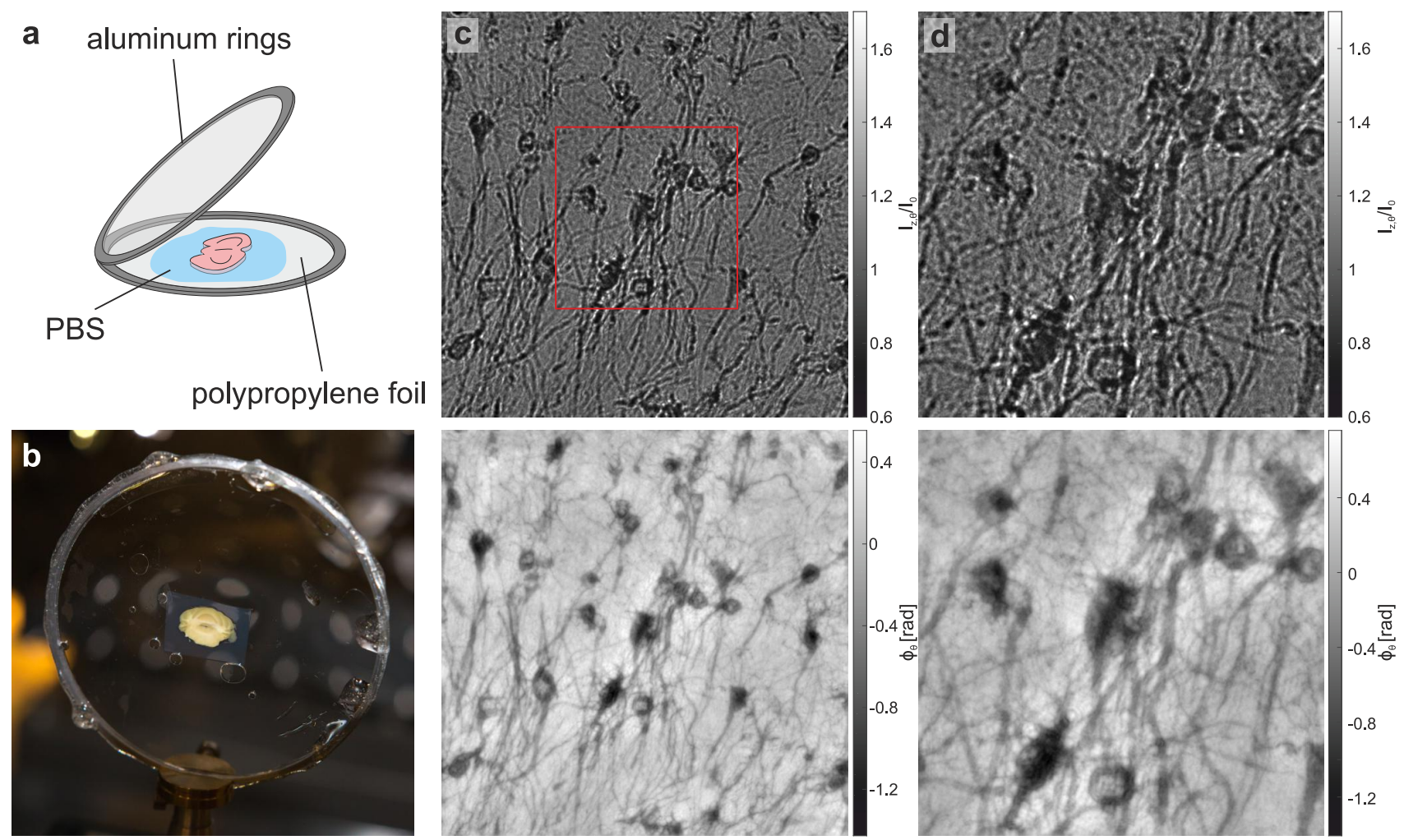

Figure 8. Hydrated Golgi-Cox stained brain slice, scanned at the P10 beamline. a Sketch of the sample mounting. To maintain environmental conditions, the sample is stored in a liquid chamber consisting of two aluminum rings which are equipped with polypropylene foil on one side. A closed tomography-compatible chamber is obtained by gluing the aluminum rings together. b Image of a brain slice stored in such a liquid chamber. This can then be mounted on a sample holder for tomographic scans. c Projection of the sample at lower magnification with an effective pixel size of $182 \mathrm{~nm}$. At the top, the flatfield-corrected projection is shown whereas the bottom image depicts the corresponding reconstructed phase, as obtained by the single distance CTF algorithm for a weakly absorbing object. The rectangle marks the position of the zoom dataset. c Flatfield-corrected projection of the zoom dataset, recorded with a pixel size of $81 \mathrm{~nm}$ (top). Phase retrieval has again been performed based on the single distance CTF algorithm (bottom).

the same position in the volume and the part of the overview scan corresponding to the zoom dataset is shown in higher magnification (Fig. 9a, left). The benefit of the higher resolution of the zoom dataset is clearly visible as the edges of the cell are sharper and the inner cell structure is reconstructed in higher detail. Furthermore, small parts of the cell which are not resolved in the overview scan, can be observed in the zoom dataset (see arrow in Fig. 9b). Fringes around the cell bodies are caused by an imperfect phase retrieval as the weakly absorbing object assumption is not fulfilled due to the high absorption of silver and and as only one propagation distance was used for reconstruction. The white halo is again identified as a missing wedge artifact due to the complete absorption of the x-rays when passing the aluminum rings.

To obtain a better impression of the three-dimensional shape of the stained cells, a (semi-)automatic segmentation of selected cells is performed (Fig. 9c and d). Same color indicates a connection between the cells, either functional or just locally due to proximity of the cells, according to the gray value based segmentation algorithm, segmenting voxels which are combined in $3 \mathrm{D}$ and lie in a specified gray value range. Again some parts which seem to belong to the cell itself or a connected neighboring cell are added manually. Note, however, that the automatic segmentation worked much better in this case, compared to the Epon embedded sample, and only a few connections had to be segmented by hand. The cells are resolved in high detail both in the overview scan and the zoom dataset. Importantly, not only the axons but also the dendrites of the neurons are visible. The benefit of the higher resolution of the zoom dataset becomes particularly evident, as more and finer dendrites can be segmented automatically or by visual inspection (see also Video 4). This is an important aspect in view 

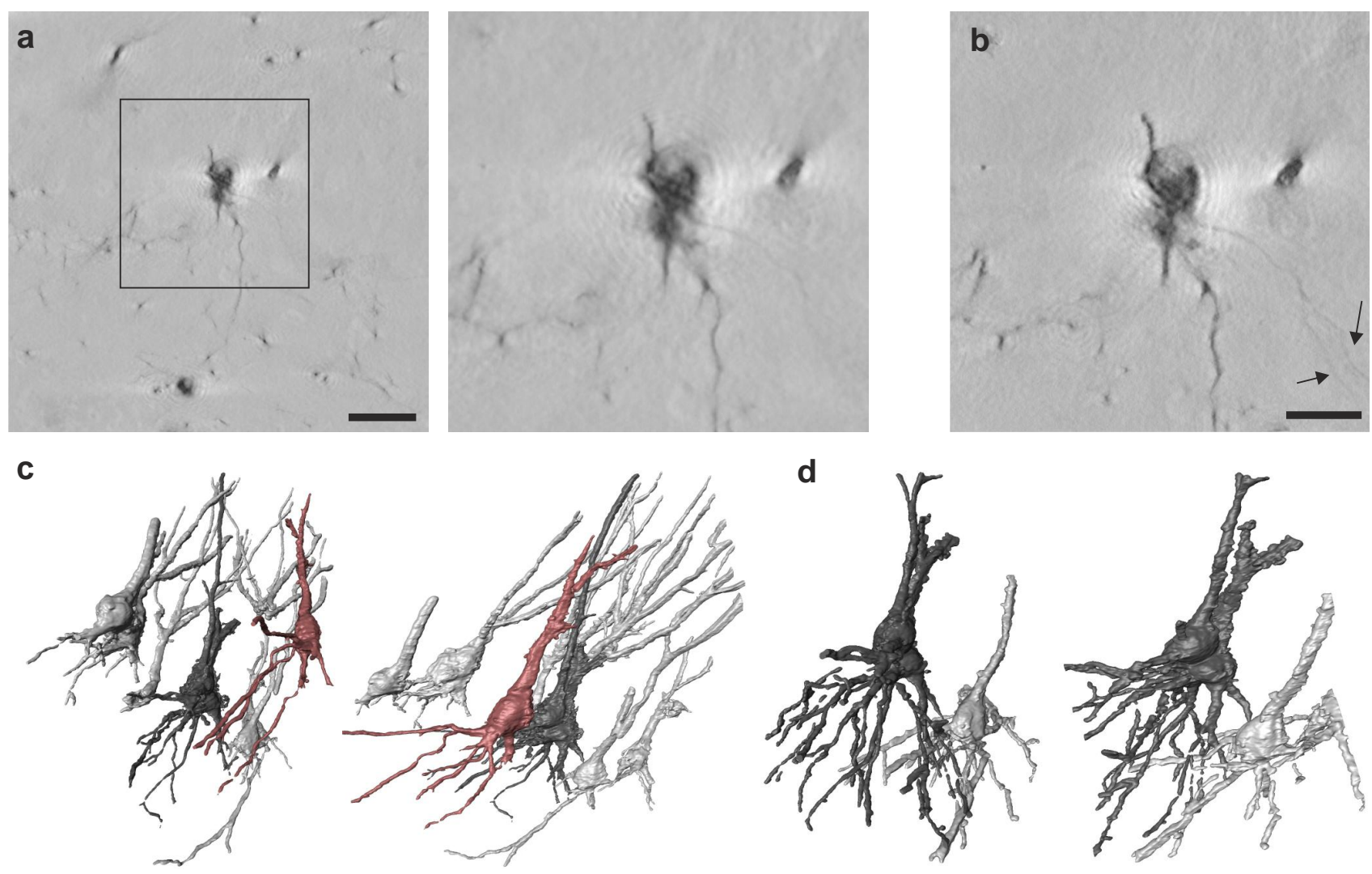

Figure 9. 3D visualization of the hydrated brain tissue. a Slice through the reconstructed volume (left). The rectangle indicates the position of the zoom dataset. A zoom of this part of the slice is shown at the right. b Corresponding slice through the reconstruction of the zoom dataset, showing the cell in greater detail as, e.g., indicated by the arrows. c Automatic volume rendering of some exemplary cells in the reconstruction. Same color means that these cells are presumably connected at some point. d (Semi)-automatic volume rendering of the corresponding cells in the zoom dataset, revealing the structure of the cell in greater detail. Some connections were not rendered automatically and therefore added by hand. Scalebars: a $50 \mu \mathrm{m}$ and $\mathbf{b} 25 \mu \mathrm{m}$

of addressing questions of neuronal connectivity in 3D.

\section{DISCUSSION}

By combination of suitable preparation protocols and phase-retrieval algorithms we have demonstrated single cell sensitivity in relatively large volumes, both with synchrotron and laboratory tomography. Comparing both results, we saw that the the high resolution synchrotron setup does not necessarily yield more detailed reconstructions of a single cell, since the effective resolution and quality is also determined by the sample preparation. In fact, results point to an incomplete staining in the Epon embedded sample, which could be caused either by the staining procedure itself or by a leakage of bound silver during the embedding procedure. While the staining is still high enough to represent axons and cell body, smaller dendrites are not visualized. Since this obviously applies to both recordings, the shape of the reconstructed neurons then appears to be very similar for the synchrotron and laboratory setup. Notwithstanding a certain lack of resolution due to the staining process, the results still clearly demonstrate that issues of neuron distribution, shape, orientation, and spatial arrangement can already be addressed with advanced laboratory phase-contrast tomography, see for example the results presented in Fig. 3.

Contrarily, the results of the hydrated brain slice illustrate the superior resolution and image quality which can be obtained with the synchrotron nano-focus setup. A significantly higher number of dendrites can now be visualized, as in this case the high resolution of the instrument is matched with a more complete staining. In this 

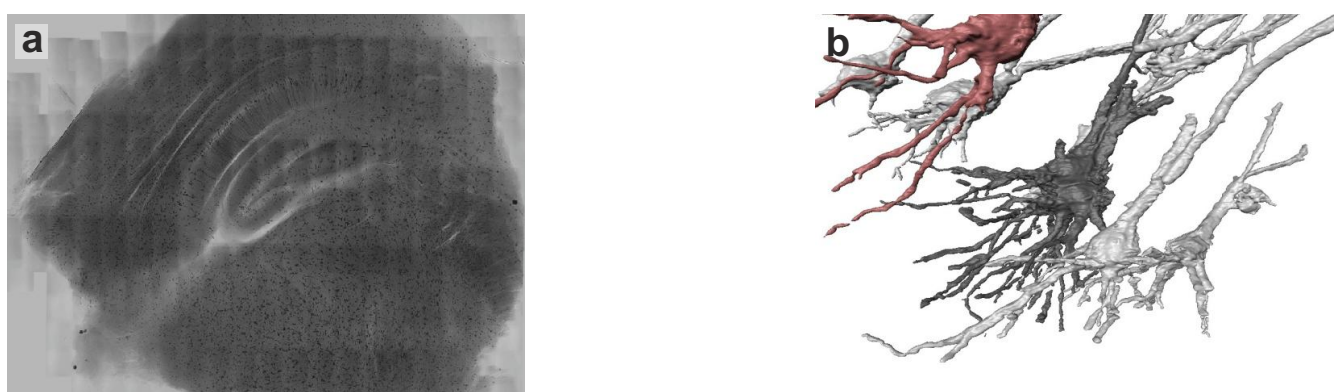

Figure 10. a Video 3: Mesh of the whole hydrated Golgi-Cox stained brain slice with a zoom to the position of the overview scan. http://dx.doi.org/10.1117/12.2238496.3 b Video 4: 3D visualization of the hydrated Golgi-Cox stained sample. As for the Epon embedded sample, the transverse slices through the volume are shown for both datasets, the overview scan and the zoom measurement. Additionally, a segmentation of the most prominent cells in the reconstructed volume is shown where same color indicates a connection between the cells. http://dx.doi.org/10.1117/12.2238496.4

way, far more details of the cell become visible. This level of detail, as represented by the reconstructions shown in Fig. 9, would probably not be resolvable by the laboratory setup, even if the hydrated tissues could be kept stable for long acquisition times. This is an important issue for laboratory tomographic scans, and we have also shown here how temperature drifts and sample motion can be corrected for. Unfortunately, the corrections are presently restricted to center-of-mass motion. The procedure may be generalized to also account for rotational drift, but internal displacements expected for hydrated tissues during long scans have to be approached by a different method. Therefore, the important question of neuronal connectivity will certainly require synchrotron radiation. To this end, a future proof-of-principle investigation should address, whether and under which conditions synapses can be identified even in unstained tissues, and traced back to the respective cell bodies. Note that for connectivity issues, the dense network has to be imaged, and hence the sparse staining according to Golgi-Cox is not very suitable, as it stains selected neurons in a statistical manner. In fact, the merit of this method is precisely in highlighting single neurons against the background.

In view of the difficulties in developing suitable staining techniques for connectivity issues, one may wonder whether one cannot circumvent staining altogether, and use only the density contrast of the native tissue. To provide a answer, at least in preliminary terms, we have imaged an unstained hydrated brain slice from the region of the cerebellum of a wild-type mouse. The experiment was performed at the P10 beamline at DESY
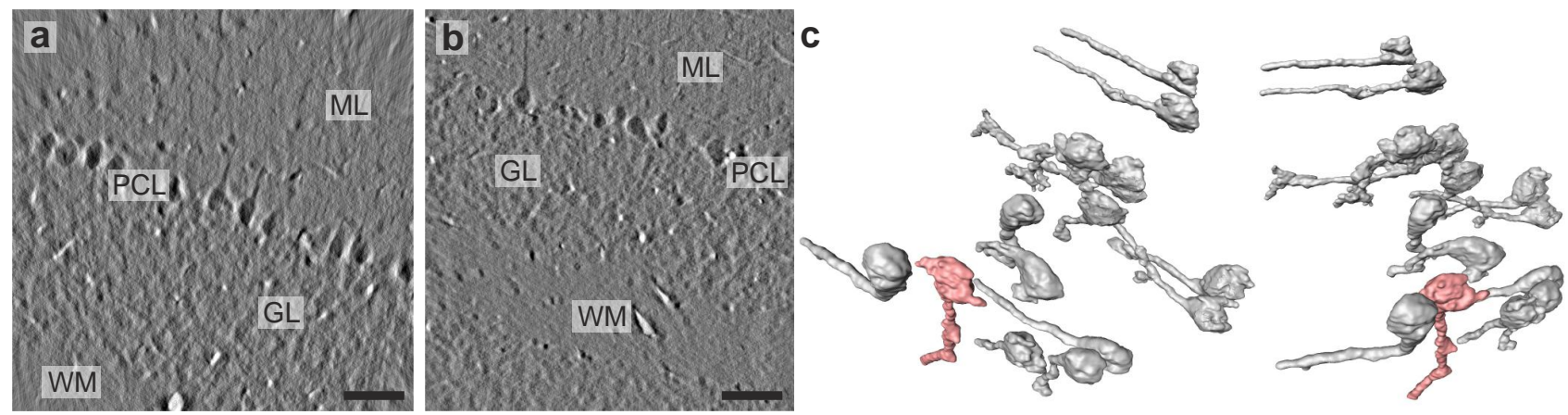

Figure 11. 3D visualization of the measurements on a hydrated unstained slice of a mouse cerebellum at the P10 beamline with a pixel size of $178 \mathrm{~nm}$. For the single distance tomogram 1663 projections over $180^{\circ}$ were recorded in a continuous scanning mode leading to a total scan time of approximately $400 \mathrm{~s}$. The phase was reconstructed using the single distance CTF algorithm with the assumption of a pure phase object due to the low absorbance. a Transversal slice through the reconstructed volume. The different layers of the cerebellum are clearly visible: white matter (WM), granular layer (GL), molecular layer (ML) and Purkinje cell layer (PCL). To some extent even the axons of the Purkinje cells are resolved. $\mathbf{b}$ Longitudinal slice thorugh the same volume, again showing the typical layers of the cerebellum. c Segmentation of some exemplary cells from the Purkinje cell layer, showing the cell bodies and to some extent the axons of the cells. The cell denoted in light red is the cell in the upper left corner in b. Scalebars: $50 \mu \mathrm{m}$ 
with a photon energy of $8 \mathrm{keV}$, promising a better interaction and therefore contrast of the unstained material compared to $13.8 \mathrm{keV}$, and a tapered silicon waveguide with air filled channels. ${ }^{30}$ The result is shown in Fig. 11. The contrast within the hydrated tissue is clearly sufficient to resolve single cells or at least the cell body of the single cells and also to some extent the axons of the slightly larger Purkinje cells. However, even with this relatively high tissue contrast, inter-cell connections cannot be resolved despite the low energy and the relatively large propagation distance, both geared towards increased contrast. Therefore, we must conclude that the use of contrast-enhancing techniques is imperative for high resolution imaging of mouse brain, as the native contrast in hydrated tissue is not sufficient to resolve single cells with the resolution needed to address the issue of neuronal connectivity. Embedding the tissue in materials with a different electron density than water, e.g., paraffin ${ }^{31}$ or, as shown here, staining with metallic ions might be a decisive step in sample preparation in order to have the sufficient resolution and contrast to decipher neuronal connectivity with the help of x-ray phase-contrast tomography. To this end, specific staining procedures should be considered, as for example the immunogold labeling already used in x-ray diffraction ${ }^{32}$ and soft $\mathrm{x}$-ray microscopy, ${ }^{33}$ staining of lipids with osmium, ${ }^{34,35}$ a standard protocol in transmission electron microscopy, as well as adaptations of protocols developed for bulk staining of FIB-SEM brain imaging. ${ }^{4}$

\section{Acknowledgments}

We thank Eleonora Carboni for help in sample preparation, Michael Sprung, Markus Osterhoff and Malte Vassholz for assistance during the beamtimes at P10 and Willem Jan Palenstijn for excellent cooperation regarding the ASTRA tomography toolbox. This work was supported by the Cluster of Excellence 171 Nanoscale Microscopy and Molecular Physiology of the Brain, the Collaborative Research Center 755 Nanoscale Photonic Imaging of the German science foundation (DFG) .

\section{REFERENCES}

[1] Amunts, K., Lepage, C., Borgeat, L., Mohlberg, H., Dickscheid, T., Rousseau, M.-É., Bludau, S., Bazin, P.-L., Lewis, L. B., Oros-Peusquens, A.-M., Shah, N. J., Lippert, T., Zilles, K., and Evans, A. C., "Bigbrain: An ultrahigh-resolution 3d human brain model," Science 340(6139), 1472-1475 (2013).

[2] Dodt, H.-U., Leischner, U., Schierloh, A., Jährling, N., Mauch, C. P., Deininger, K., Deussing, J. M., Eder, M., Zieglgänsberger, W., and Becker, K., "Ultramicroscopy: three-dimensional visualization of neuronal networks in the whole mouse brain," Nature methods 4(4), 331-336 (2007).

[3] Dorr, A., Lerch, J. P., Spring, S., Kabani, N., and Henkelman, R. M., "High resolution three-dimensional brain atlas using an average magnetic resonance image of 40 adult c57bl/6j mice," Neuroimage 42(1), 60-69 (2008).

[4] Mikula, S., Binding, J., and Denk, W., "Staining and embedding the whole mouse brain for electron microscopy," Nature methods 9(12), 1198-1201 (2012).

[5] Stampanoni, M., Mokso, R., Marone, F., Vila-Comamala, J., Gorelick, S., Trtik, P., Jefimovs, K., and David, C., "Phase-contrast tomography at the nanoscale using hard x rays," Phys. Rev. B 81(14), 140105 (2010).

[6] Weitkamp, T., Diaz, A., David, C., Pfeiffer, F., Stampanoni, M., Cloetens, P., and Ziegler, E., "X-ray phase imaging with a grating interferometer," Opt. Express 13(16), 6296-6304 (2005).

[7] Schulz, G., Weitkamp, T., Zanette, I., Pfeiffer, F., Beckmann, F., David, C., Rutishauser, S., Reznikova, E., and Müller, B., "High-resolution tomographic imaging of a human cerebellum: comparison of absorption and grating-based phase contrast," J. R. Soc. Interface 7(53), 1665-1676 (2010).

[8] Munro, P. R., Ignatyev, K., Speller, R. D., and Olivo, A., "Phase and absorption retrieval using incoherent x-ray sources," Proceedings of the National Academy of Sciences 109(35), 13922-13927 (2012).

[9] Hagen, C., Munro, P., Endrizzi, M., Diemoz, P., and Olivo, A., "Low-dose phase contrast tomography with conventional x-ray sources," Medical physics 41(7), 070701 (2014).

[10] Zanette, I., Zhou, T., Burvall, A., Lundström, U., Larsson, D. H., Zdora, M., Thibault, P., Pfeiffer, F., and Hertz, H. M., "Speckle-based x-ray phase-contrast and dark-field imaging with a laboratory source," Phys. Rev. Lett. 112(25), 253903 (2014). 
[11] Cloetens, P., Ludwig, W., Baruchel, J., Van Dyck, D., Van Landuyt, J., Guigay, J. P., and Schlenker, M. a., "Holotomography: Quantitative phase tomography with micrometer resolution using hard synchrotron radiation x rays," Appl. Phys. Lett. 75(19), 2912-2914 (1999).

[12] Lagomarsino, S., Cedola, A., Cloetens, P., Di Fonzo, S., Jark, W., Soullie, G., and Riekel, C., "Phase contrast hard x-ray microscopy with submicron resolution," Appl. Phys. Lett. 71(18), 2557-2559 (1997).

[13] Paganin, D. and Nugent, K. A., "Noninterferometric Phase Imaging with Partially Coherent Light," Phys. Rev. Lett. 80, 2586-2589 (1998).

[14] Bartels, M., Krenkel, M., Haber, J., Wilke, R. N., and Salditt, T., "X-ray holographic imaging of hydrated biological cells in solution," Phys. Rev. Lett. 114, 048103 (2015).

[15] Paganin, D. M., [Coherent X-Ray Optics], New York: Oxford University Press (2006).

[16] Groso, A., Abela, R., and Stampanoni, M., "Implementation of a fast method for high resolution phase contrast tomography," Opt. Express 14(18), 8103-8110 (2006).

[17] Witte, Y. D., Boone, M., Vlassenbroeck, J., Dierick, M., and Hoorebeke, L. V., "Bronnikov-aided correction for x-ray computed tomography," J. Opt. Soc. Am. A 26(4), 890-894 (2009).

[18] Turner, L., Dhal, B., Hayes, J., Mancuso, A., Nugent, K., Paterson, D., Scholten, R., Tran, C., and Peele, A., "X-ray phase imaging: Demonstration of extended conditions for homogeneous objects," Opt. Express 12(13), 2960-2965 (2004).

[19] Zabler, S., Cloetens, P., Guigay, J.-P., Baruchel, J., and Schlenker, M., "Optimization of phase contrast imaging using hard x rays," Rev. Sci. Instrum. 76(7), 073705 (2005).

[20] Bartels, M., Hernandez, V. H., Krenkel, M., Moser, T., and Salditt, T., "Phase contrast tomography of the mouse cochlea at microfocus x-ray sources," Appl. Phys. Lett. 103(8), 083703 (2013).

[21] Kalbfleisch, S., Neubauer, H., Krüger, S. P., Bartels, M., Osterhoff, M., Mai, D. D., Giewekemeyer, K., Hartmann, B., Sprung, M., and Salditt, T., "The Göttingen Holography Endstation of Beamline P10 at PETRA III/DESY," AIPConf.Proc. 1365(1), 96-99 (2011).

[22] Kalbfleisch, S., Osterhoff, M., Giewekemeyer, K., Neubauer, H., Kruger, S. P., Hartmann, B., Bartels, M., Sprung, M., Leupold, O., Siewert, F., and Salditt, T., "The holography endstation of beamline P10 at PETRA III," SRI 2009, 10TH INTERNATIONAL CONFERENCE ON RADIATION INSTRUMENTATION 1234(1), 433-436 (2010).

[23] Salditt, T., Osterhoff, M., Krenkel, M., Wilke, R. N., Priebe, M., Bartels, M., Kalbfleisch, S., and Sprung, M., "Compound focusing mirror and X-ray waveguide optics for coherent imaging and nano-diffraction," $J$. Synchrotron Rad. 22(4), 867-878 (2015).

[24] Krüger, S. P., Giewekemeyer, K., Kalbfleisch, S., Bartels, M., Neubauer, H., and Salditt, T., "Sub-15 nm beam confinement by twocrossed x-ray waveguides," Opt. Express 18(13), 13492-13501 (2010).

[25] Krüger, S. P., Neubauer, H., Bartels, M., Kalbfleisch, S., Giewekemeyer, K., Wilbrandt, P. J., Sprung, M., and Salditt, T., "Sub-10 $\mathrm{nm}$ beam confinement by X-ray waveguides: design, fabrication and characterization of optical properties," J. Synchrotron Rad. 19(2), 227-236 (2012).

[26] Homann, C., Hohage, T., Hagemann, J., Robisch, A.-L., and Salditt, T., "Validity of the empty-beam correction in near-field imaging," Phys. Rev. A 91, 013821 (2015).

[27] Palenstijn, W., Batenburg, K., and Sijbers, J., "Performance improvements for iterative electron tomography reconstruction using graphics processing units (gpus)," Journal of structural biology 176(2), 250-253 (2011).

[28] van Aarle, W., Palenstijn, W. J., De Beenhouwer, J., Altantzis, T., Bals, S., Batenburg, K. J., and Sijbers, J., "The astra toolbox: A platform for advanced algorithm development in electron tomography," Ultramicroscopy 157, 35-47 (2015).

[29] Krenkel, M., Markus, A., Bartels, M., Dullin, C., Alves, F., and Salditt, T., "Phase-contrast zoom tomography reveals precise locations of macrophages in mouse lungs," Sci. Rep. 5, 09973 (2015).

[30] Chen, H.-Y., Hoffmann, S., and Salditt, T., "X-ray beam compression by tapered waveguides," Applied Physics Letters 106(19), 194105 (2015).

[31] Hieber, S. E., Bikis, C., Khimchenko, A., Schweighauser, G., Hench, J., Chicherova, N., Schulz, G., and Müller, B., "Tomographic brain imaging with nucleolar detail and automatic cell counting," Scientific Reports 6 (2016). 
[32] Nelson, J., Huang, X., Steinbrener, J., Shapiro, D., Kirz, J., Marchesini, S., Neiman, A. M., Turner, J. J., and Jacobsen, C., "High-resolution x-ray diffraction microscopy of specifically labeled yeast cells," PNAS 107(16), 7235-7239 (2010).

[33] Sakdinawat, A. and Attwood, D., "Nanoscale x-ray imaging," Nature photonics 4(12), 840-848 (2010).

[34] Lareida, A., Beckmann, F., Schrott-Fischer, A., Glueckert, R., Freysinger, W., and Müller, B., "Highresolution x-ray tomography of the human inner ear: synchrotron radiation-based study of nerve fibre bundles, membranes and ganglion cells," Journal of microscopy 234(1), 95-102 (2009).

[35] Bartels, M., Krenkel, M., Cloetens, P., Möbius, W., and Salditt, T., "Myelinated mouse nerves studied by x-ray phase contrast zoom tomography," J. of Structural Biology, - (2015). 